\title{
Cavity Nucleation Study during Quasi- Superplasticity of an Ultralight Coarse-Grained Rolled Mg-7.28Li-2.19Al-0.1Y Alloy
}

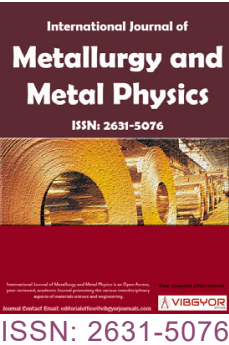

Furong $\mathrm{CaO}^{1^{*}}$, Bijin Zhou', Nanpan Guo' and Jinrui Liang'

${ }^{1}$ School of Materials Science and Engineering, Northeastern University, PR China

${ }^{2}$ School of Mechanical Engineering, Suzhou University of Science and Technology, PR China

\begin{abstract}
Cavity or cavitation is a phenomenon in superplasticity and creep and is significant to the prediction of cavity mechanism. However, compared to cavity growth, cavity nucleation is rarely reported in magnesium alloy, especially Mg-Li alloy. To investigate the cavitation nucleation, a novel Mg-7.28Li-2.19Al-0.1Y alloy has been fabricated by hot rolling and cold rolling; its flow stress and microstructural cavitation at elevated temperatures were investigated by optical microscopy and tensile tester. The maximum elongation to failure of $265.8 \%$ was demonstrated in this alloy at a temperature of $623 \mathrm{~K}$ and a strain rate of $5.0 \times 10^{-4}$ $\mathrm{s}^{-1}$. Experimental results revealed that cavity or cavitation nucleated at interphase boundary and $\alpha-\mathrm{Mg}$ grain boundary in quasi-superplasticity Mg-7.28Li-2.19Al-0.091Y alloy. The present alloy had coarse grain sizes and cavity nucleation was easy to initiate in this coarse-grained alloy. Helmholtz free energy map was plotted to predict the easy and difficulty of cavity nucleation in the present alloy. This work will enhance the workability and understand the fracture initiation behavior in the present $\alpha-\mathrm{Mg}$ phase dominated alloy.
\end{abstract}

\section{Keywords}

Magnesium, Cavitation nucleation, Quasi-superplasticity, Flow stress, Microstructure

\section{Introduction}

Magnesium-lithium (Mg-Li) alloy is the lightest nonpoisonous alloy. Due to extremely low density, excellent specific stiffness, good weight-to-density ratio, good electromagnetic shielding property, and damping property, studies on Mg-Li alloys have attracted extensive attention from the researchers, not only for room temperature property and microstructure [1-3] but also for high temperature behavior, in particular, superplasticity [4-9]. Under this background, we designed and fabricated a novel two-phase Mg-7Li-2Al-1Y alloy and investigated its high temperature behavior.

Superplasticity reflects the capability of materials to exhibit exceptional ductility or elongation, typically several hundred, and occasionally thousands [10]. Superplastic forming can realize the forming of the complex components such as thin wall high rib components under a small load. During superplasticity study, extensive researches are devoted to the high temperature flow deformation mechanism. In the meantime, cavity or cavitation study also has carried out the limited investigation.

*Corresponding author: Furong Cao, School of Materials Science and Engineering, Northeastern University, Shenyang 110819, PR China

Accepted: December 29, 2021; Published: December 31, 2021

Copyright: (C) 2021 Cao F, et al. This is an open-access article distributed under the terms of the Creative Commons Attribution License, which permits unrestricted use, distribution, and reproduction in any medium, provided the original author and source are credited.

Cao et al. Int J Metall Met Phys 2021, 6:071

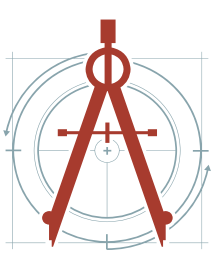


Cavity or cavitation nucleation, growth, coalescence, and interlinkage fracture reflect different stages of fracture. Cavity or cavitation phenomenon originated from the report in Zn-22Al superplasticity [11]. In recent years, cavity growth has been moderately studied in oxygen free copper [12], 5A70 aluminum alloy [13], 5083 aluminum alloy [14], Al-4.7Mg-Mn-Fe alloy [15], Zn-22Al zinc-based alloy [16], and so forth. However, compared to the cavity growth study, cavity nucleation is rarely reported. To the best of our knowledge, only a few reports are devoted to the experimental study of superplastic Mg-Li- $\mathrm{Zn}$ cavity growth $[17,18]$, and no information is available studying on the cavity nucleation behavior of $\mathrm{Mg}$ 7Li-2Al-1Y alloy designed and processed by rolling. Thus, it is necessary to investigate the flow stress and microstructure cavitation through hot tensile tests and microstructural characterization. Meanwhile, aiming at the facts of only limited experimental researches on cavity phenomenon recently, it is necessary to use our cavitation nucleation model to plot the free energy map to depict the variation in free energy with cavity radius in this new alloy.

In this work, our investigation contents include three aspects: (i) A novel Mg-7.28Li-2.19Al-0.1Y alloy has been fabricated by hot rolling and cold rolling; (ii) Its flow stress and microstructural cavitation at elevated temperatures were investigated; (iii) The free energy map was plotted to analyze the cavitation nucleation mechanism and corroborated in this alloy.

\section{Experimental Procedures}

Raw materials $\mathrm{Mg}$, Al, and Li (purity more than 99.9 wt.\%) and Al-30 wt.\% Y master alloy were melted in an electric furnace with a flux of $\mathrm{LiCl}$ and $\mathrm{LiF}(3: 1)$ under the protection of argon (Ar) atmosphere, as detailed in elsewhere [19]. The melt was cast in a water circulated copper mold to obtain an Mg-7Li-2Al$1 Y$ alloy (wt.\%) ingot. The analyzed chemical composition of the ingot was Mg-7.28Li-2.19Al-0.1Y. After homogenization at $473 \mathrm{~K}$ for $20 \mathrm{~h}$, the ingot was hot rolled at $573 \mathrm{~K}$ to a plate $5 \mathrm{~mm}$ in thickness with a percent reduction of $77.3 \%$ and cold rolled to a sheet $2 \mathrm{~mm}$ in thickness with a percent reduction of $60 \%$. The annealing system was $623 \mathrm{~K}$ for $3 \mathrm{~h}$. Dog-bone samples with dimensions of $10 \mathrm{~mm}$ in gauge length, 6 $\mathrm{mm}$ in width, and $2 \mathrm{~mm}$ in thickness whose direction was parallel to rolling direction were stamped on a hydraulic press. Tensile tests were conducted on CMT5105 microcomputer controlled electronic universal tester at temperatures of $423-623 \mathrm{~K}$ at initial strain rates of $1.62 \times 10^{-2}-1.67 \times 10^{-4} \mathrm{~s}^{-1}$. Samples for cavity or cavitation observation were polished by conventional metallographic methods and etched in a solution of $10 \% \mathrm{HCl}+90 \% \mathrm{EtOH}$. The etched samples were observed on Olympus DSX500 optical microscope. The grain size was measured by Image-Pro Plus (IPP) software.

\section{Our Cavity Nucleation Model}

Helmholtz free energy model of cavity nucleation is given by the following formula according to our report on typical superplasticity of Al-Si-Mg alloy [20]:

$$
\Delta G=-r^{3} F_{v}(\alpha) \sigma_{P}+r^{2}\left[\gamma F_{S}(\alpha)-\gamma F_{b}(\alpha)\right]-r^{3} F_{V}^{\prime}(\alpha) \sigma_{P}^{2} / 2 E
$$

where $r$ is the cavity radius, the first term is the work done by the applied stress on the system, the second and third terms are the change in surface and grain boundary areas, respectively and hence energies, and the fourth term is the change of elastic strain energy in the system. $F_{v^{\prime}} F_{\mathrm{b}}$ and $F_{\mathrm{s}}$ are dimensionless functions which when they are multiplied by $r^{3}$ and $r^{2}$, give the volume, the surface area and the grain boundary area consumed by the cavity, respectively. $F_{v}^{\prime}$ multiplied by $r^{3}$ gives the volume within which the elastic strain energy is relaxed due to the formation of the cavity, $\sigma_{p}$ is the pile-up stress of dislocations, and $E$ is Young's modulus.

Through derivation, one gets

$$
\Delta G=-2.53\left(\frac{2}{3} \times \frac{d}{1.74 b} \times \frac{\sigma^{2}}{G}\right) r^{3}+[9.31 \gamma-0.5 \times 2.93 \gamma] r^{2}-3.79 \times \frac{\left(\frac{2}{3} \times \frac{d}{1.74 b} \times \frac{\sigma^{2}}{G}\right)^{2}}{2 E} r^{3}
$$

where $d=1.74 L$, here $L$ is the linear intercept grain size or the length of dislocation pile-up, $\sigma$ is the applied stress, $b$ is the magnitude of Burgers vector, and $G$ is the shear modulus, $E=2 G(1+v)$ [21], here, 
$v$ is Poisson's ratio.

Differentiate Eq. (2), let $\frac{\partial \Delta G}{\partial r}=0$, the critical radius of cavity nucleation, $r_{c}$, is obtained:

$$
r_{c}=\frac{\gamma}{\sigma}\left[\frac{40.95}{7.89+2.18\left(\frac{d}{b}\right)\left(\frac{\sigma}{G}\right)\left(\frac{\sigma}{E}\right)\left(\frac{d}{b}\right)\left(\frac{\sigma}{G}\right)}\right]
$$

Further, Eq. (3) was simplified into the following form:

$$
r_{c}=5.2\left(\frac{\gamma}{\sigma}\right)\left(\frac{b}{d}\right)\left(\frac{G}{\sigma}\right)
$$

\section{Results and Discussion}

\section{Flow stress curves and microstructural cavitation morphology}

Figure 1 presents the true stress - strain curves of Mg-7.28Li-2.19Al-0.091Y alloy at different tensile temperatures at the same initial strain rate. For different flow stress curves, the flow stress level decreases with increasing temperature and/or decreasing strain rate. That is because with the increase in temperature, thermal activation and atomic diffusion accelerate, dislocation density decreases, and flow stress decreases; with the decrease in strain rate, the tensile deformation time prolongs, the alloy has sufficient time to promote thermal activation and atomic diffusion, dislocation density decreases, and flow stress decreases. For single flow stress curve, the curves exhibit single peak form and are typical dynamic recrystallization curves. Due to dynamic recrystallization, after the peak stress, the flow stress exhibits a slow decreasing true stress with increasing strain, i.e., strain softening. As shown in Figure $1 \mathrm{c}$, at a temperature of $623 \mathrm{~K}$ and a strain rate of $5.0 \times 10^{-4} \mathrm{~s}^{-1}$ the true strain of 1.297 corresponds to the elongation to failure of $265.8 \%$ according to the relation: true strain=In (1+engineering strain or elongation), exhibiting quasi-superplasticity.
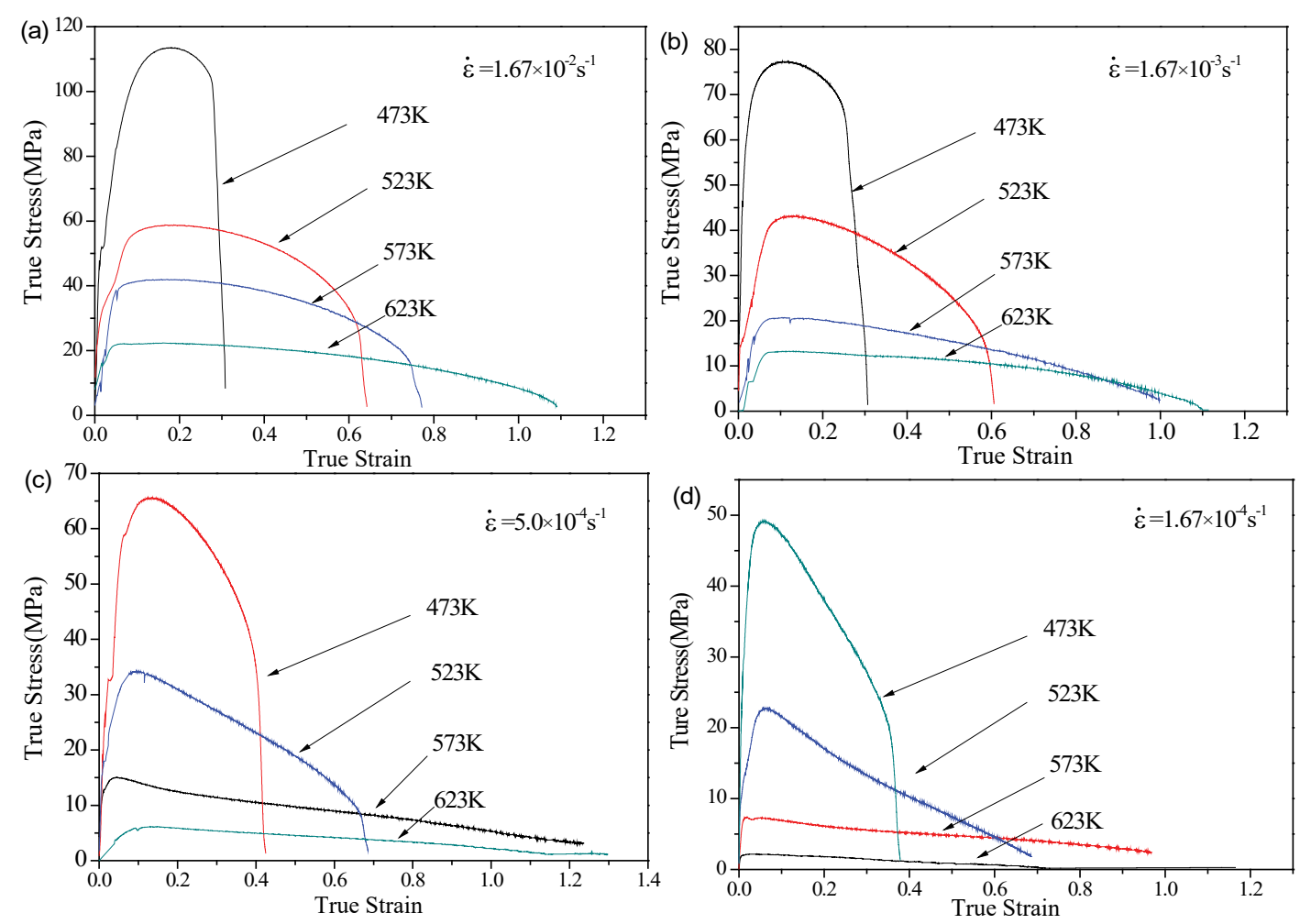

Figure 1: True stress - strain curves of Mg-7.28Li-2.19Al-0.091Y alloy at different tensile temperatures at the same initial strain rate: (a) $1.67 \times 10^{-2} \mathrm{~s}^{-1}$; (b) $1.67 \times 10^{-3} \mathrm{~s}^{-1}$; (c) $5 \times 10^{-4} \mathrm{~s}^{-1}$; (d) $1.67 \times 10^{-4} \mathrm{~s}^{-1}$. 


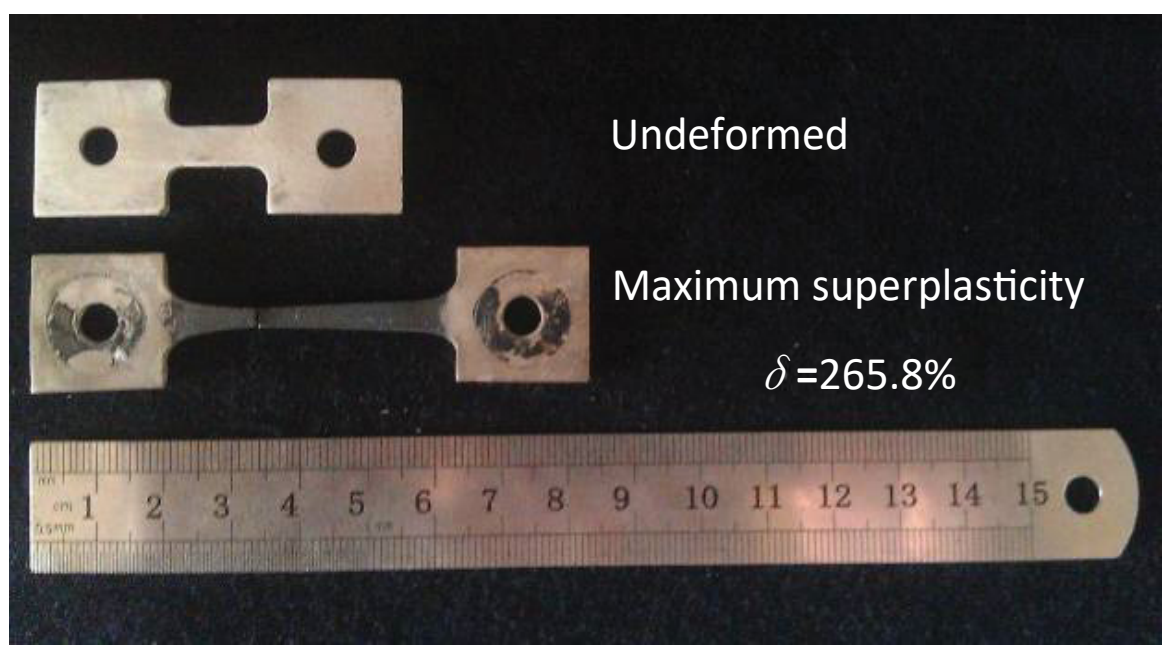

Figure 2: Specimens showing the maximum superplasticity of Mg-7.28Li-2.19Al-0.091Y alloy.
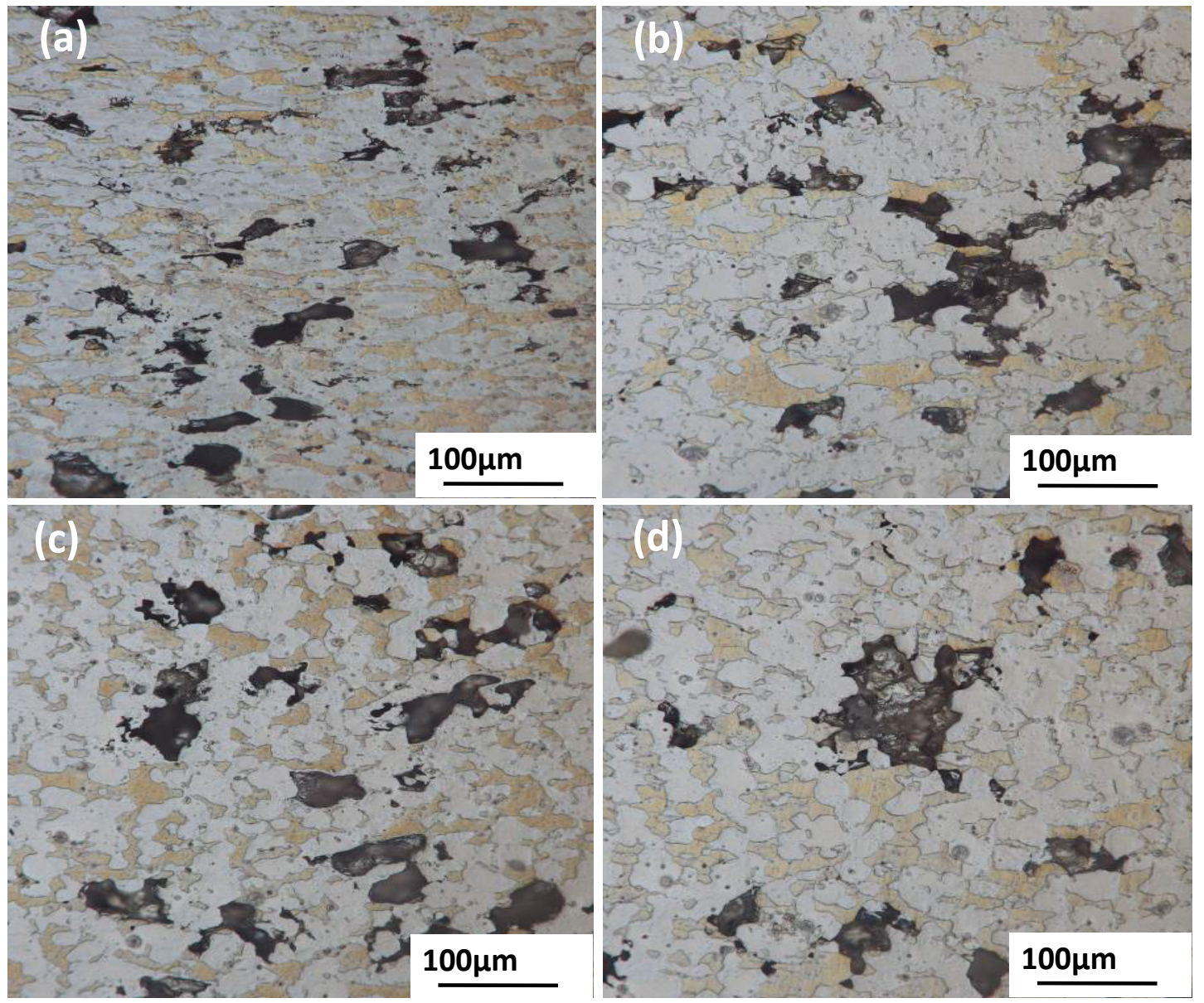

Figure 3: Cavitations in Mg-7.28Li-2.19Al-0.091Y alloy specimen after tensile test to fracture at temperature of $573 \mathrm{~K}$ with different initial strain rates and grain sizes: (a) $1.67 \times 10^{-2} \mathrm{~s}^{-1}, d=40.4 \mu \mathrm{m}$; (b) $1.67 \times 10^{-3} \mathrm{~s}^{-1}, d=35.5$ $\mu \mathrm{m}$; (c) $5 \times 10^{-4} \mathrm{~s}^{-1}, d=31.7 \mu \mathrm{m}$; (d) $1.67 \times 10^{-4} \mathrm{~s}^{-1}, d=33.4 \mu \mathrm{m}$.

Figure 2 displays the macroscopic appearance of maximum superplasticity in Mg-7.28Li-2.19Al-0.091Y alloy. The maximum elongation to failure of $265.8 \%$ is obtained at a temperature of $623 \mathrm{~K}$ and a strain rate of $5.0 \times 10^{-4} \mathrm{~s}^{-1}$.

Figure 3 shows the cavitations in Mg-7.28Li-2.19Al-0.091Y alloy specimen after tensile test to fracture 
at the temperature of $573 \mathrm{~K}$ with different initial strain rates. White grain is hexagonal closed-packed structured $\alpha-\mathrm{Mg}$ matrix. Yellow grain is body-centered cubic structured $\beta$-Li phase. Black part indicates the cavity or cavitation. The grain sizes ranging from 31.7 to $40.4 \mu \mathrm{m}$ exceed $10 \mu \mathrm{m}$, and the present alloy is a coarse-grained alloy. Hence, this alloy is a two-phase coarse-grained alloy. It is visible that cavity or cavitation nucleates at interphase $(\alpha-\mathrm{Mg} / \beta-\mathrm{Li})$ boundary and $\alpha-\mathrm{Mg}$ grain boundary. In Figure $3 \mathrm{~b}$, cavity interlinkage (long strip-like cavitation) can be seen while in Figure $3 d$, cavity coalescence can be seen. In addition, cavity distribution is not homogeneous due to the local accumulation and relaxation of stress concentration.

\section{Calculation of cavity nucleation}

To perform estimation of the critical cavity radius and plot Helmholtz free energy curves under different deformation conditions, the following experimental data and physics parameters or constants are collected. Flow stress, $\sigma$, is obtained from Figure 1 at a true strain of $0.2 ;$ Grain size, $d$, is obtained
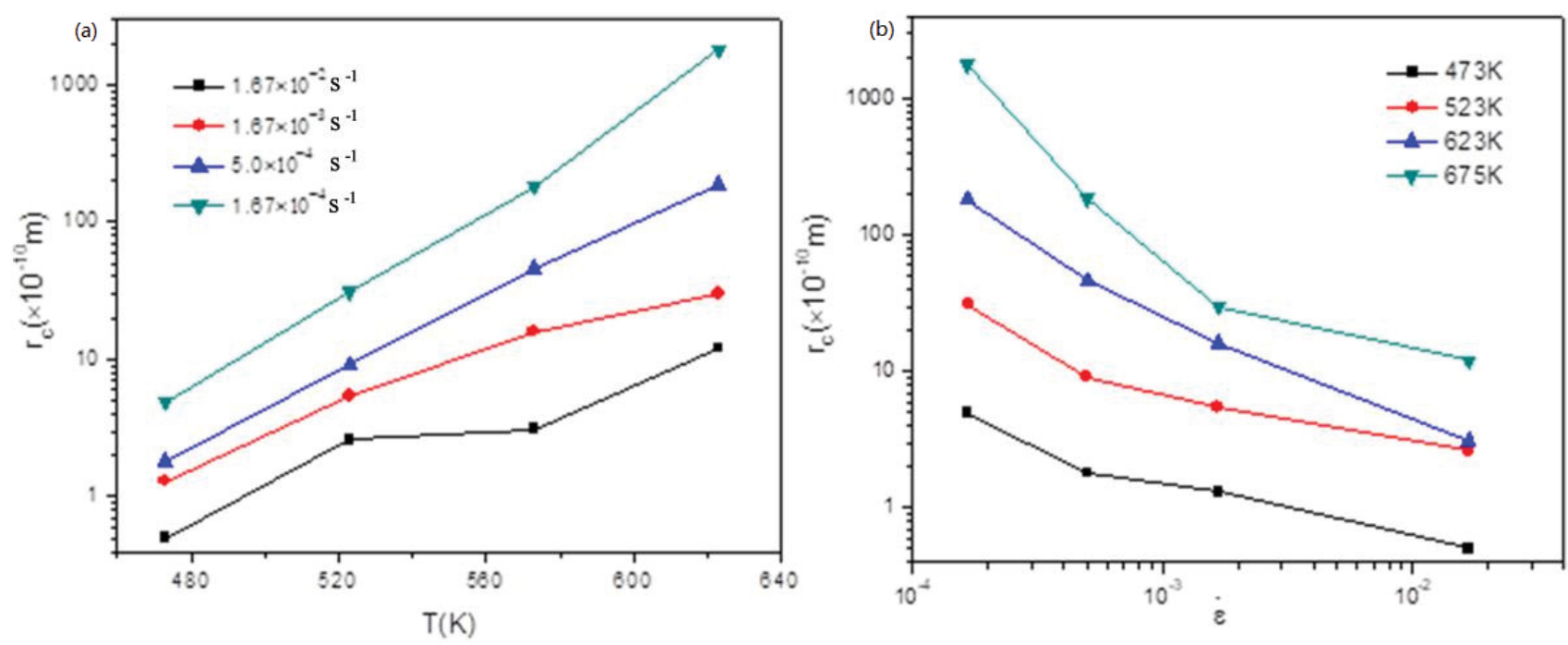

Figure 4: Critical cavity radius as a function of: (a) Deformation temperature; and (b) Strain rate in Mg-7.28Li2.19Al-0.091Y alloy deformed at temperatures of $423-623 \mathrm{~K}$ at strain rates of $1.62 \times 10^{-2}-1.67 \times 10^{-4} \mathrm{~s}^{-1}$.

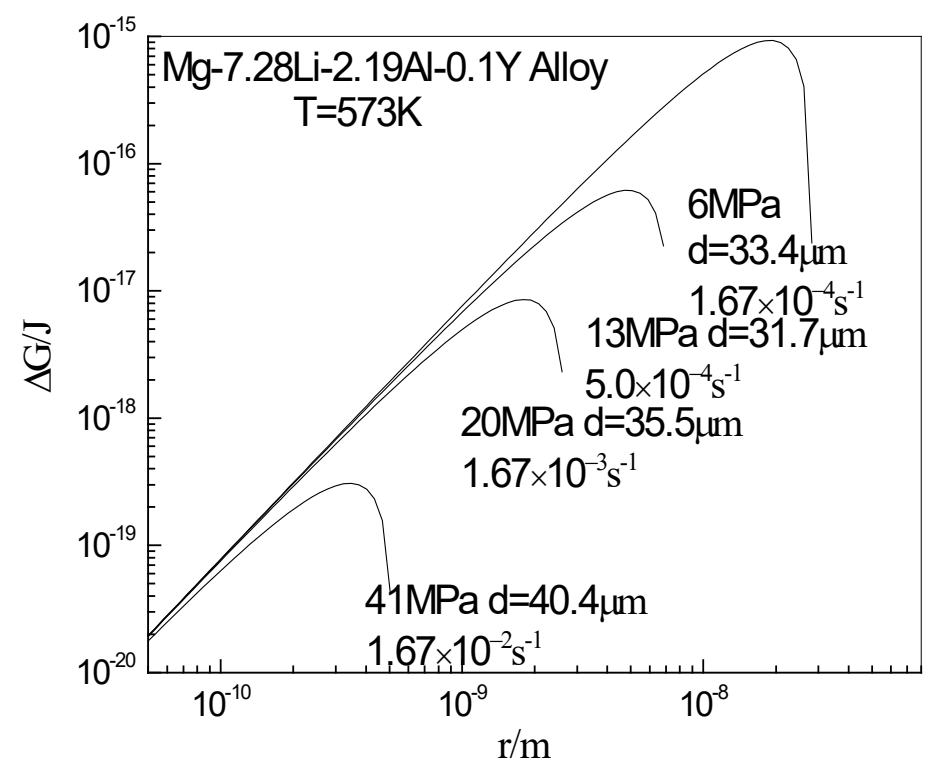

Figure 5: Helmholtz free energy as a function of cavity radius in Mg-7.28Li-2.19Al-0.091Y alloy at a temperature of $573 \mathrm{~K}$. 
from Figure 2; $v=0.28$ [22]; $b=3.21 \times 10^{-10} \mathrm{~m}$ [22]; $\nu=1 \mathrm{~J} / \mathrm{m}^{2}$ [23]; $G$ for $\mathrm{Mg}$ is given by [17]

$$
G(M P a)=1.66 \times 10^{4}[1-0.49(T-300) / 943]
$$

where the definition of $G$ and $T$ are the shear modulus and absolute temperature in Kelvin.

Figure 4 displays the critical cavity radius as a function of deformation temperature and strain rate in Mg-7.28Li-2.19Al-0.091Y alloy. It is noted that at the given strain rate, critical cavity radius increases with increasing temperature, and cavity nucleation becomes difficult; at the given temperature, critical cavity radius decreases with increasing strain rate, and cavity nucleation becomes easy.

Figure 5 displays the curve of Helmholtz free energy as a function of cavity radius in Mg-7.28Li-2.19Al$0.091 \mathrm{Y}$ alloy at a temperature of $573 \mathrm{~K}$. High flow stress leads to low peak free energy, and cavity nucleation becomes easy, whereas low flow stress leads to high peak free energy, and cavity nucleation becomes difficult. It is known from Eq. (4) that critical cavity radius, $r_{c^{\prime}}$ decreases with increasing applied stress, $\sigma$, which indicates that cavity under high applied stress is more apt to nucleation than that under low applied stress at given grain size. Meanwhile, it is known from Eq. (4) that critical cavity radius, $r_{c^{\prime}}$ decreases with increasing grain size, $d$, which indicates that coarse-grained alloy is more apt to cavity nucleation than fine-grained alloy at the given stress. The present alloy has coarse grain sizes and cavity nucleation is easy to initiate in this coarse-grained alloy, as proved by Figure 2.

\section{Conclusions}

(1) Cavity or cavitation nucleated at interphase boundary and at $\alpha$-Mg grain boundary in quasisuperplasticity Mg-7.28Li-2.19Al-0.091Y alloy subjected to rolling.

(2) Helmholtz free energy map was plotted to predict the easy and difficulty of cavity nucleation in the present alloy. The present alloy had coarse grain sizes and cavity nucleation was easy to initiate in this coarse-grained alloy.

(3) The maximum elongation to failure of $265.8 \%$ was demonstrated in this alloy at a temperature of 623 $\mathrm{K}$ and a strain rate of $5.0 \times 10^{-4} \mathrm{~s}^{-1}$.

\section{Acknowledgments}

This work is supported financially by the National Natural Science Foundation of China [Grant number 51334006].

\section{References}

1. Cao FR, Zhou BJ, Ding X, Zhang J, Xu GM (2018) Mechanical properties and microstructural evolution in a superlight Mg-7.28Li-2.19Al-0.091Y alloy fabricated by rolling. Journal of Alloys and Compounds 745: 436-445.

2. Cao FR, Zhang J, Ding X, Xue GQ, Liu SY, et al. (2019) Mechanical properties and microstructural evolution in a superlight Mg-6.4Li-3.6Zn-0.37Al-0.36Y alloy processed by multidirectional forging and rolling. Materials Science and Engineering A 760: 377-393.

3. Cao FR, Sun CF, Shang HH, Xiang C, Liu RJ (2021) Microstructure evolution and mechanical properties in an ultralight Mg-2.76Li-3AI-2.6Zn-0.39Y alloy. Materials Science and Engineering A 822: 141680.

4. Chen DX, Kong J, Gui ZZ, Li W, Long Y, et al. (2021) High-temperature superplastic behavior and ECAP deformation mechanism of two-phase Mg-Li alloy. Materials Letters 301: 130358.

5. Zhang TL, Tokunaga T, Ohno M, Wu RZ, Zhang ML, et al. (2018) Low temperature superplasticity of a dual-phase $\mathrm{Mg}-\mathrm{Li}-\mathrm{Zn}$ alloy processed by a multi-mode deformation process. Materials Science and Engineering A 737: 61-68.

6. Zhou MR, Morisada Y, Fujii H, Wang Y (2020) Pronounced low-temperature superplasticity of friction stir processed Mg-9Li-1Zn alloy. Materials Science and Engineering A 780: 139071.

7. Yang HP, Zhang X, Chen P, Fu MW, Wang GC, et al. (2019) Investigation on the enhanced maximum strain rate sensitivity $(\mathrm{m})$ superplasticity of Mg-9Li-1Al alloy by a two-step deformation method. Materials Science and Engineering A 764: 138219. 
8. Dutkiewicz J, Kalita D, Maziarz W, Faryna M (2020) Superplastic deformation of Mg-9Li-2Al-0.5Sc alloy after grain refinement by KoBo extrusion and cyclic forging. Archives of Civil and Mechanical Engineering 20: 121.

9. Cao FR, Ding H, Li YL, Zhou G, Cui JZ (2010) Superplasticity, dynamic grain growth and deformation mechanism in ultralight two-phase magnesium-lithium alloys. Materials Science and Engineering A 527: 2335-2341.

10.Langdon TG (2009) Seventy-five years of superplasticity: historic developments and new opportunities. Journal of Materials Science 44: 5998-6010.

11. Mohamed FA, Ahmed MI, Langdon TG (1977) Factors influencing ductility in the superplastic Zn-22 pct Al eutectoid. Metallurgical Transactions A 8: 933-938.

12.Sandström R, Wu R, Hagström J (2016) Grain boundary sliding in copper and its relation to cavity formation during creep. Materials Science and Engineering A 651: 259-268.

13.Li S, Jin SY, Huang ZG (2018) Cavity behavior of fine-grained 5A70 aluminum alloy during superplastic formation. Metals 8: 1065.

14.Ridley N, Bate PS, Zhang B (2007) Effect of strain rate path on cavitation in superplastic aluminium alloy. Materials Science and Engineering A 463: 224-230.

15.Jin H (2019) Optimization of aluminum alloy AA5083 for superplastic and quick plastic forming. Metallurgical and Materials Transactions A 50: 3868-3890.

16.Kawasaki M, Langdon TG (2012) An investigation of cavity development during superplastic flow in a zincaluminum alloy processed using severe plastic deformation. Materials Transactions 53: 87-95.

17.Kawasaki M, Kubota K, Higashi K, Langdon TG (2006) Flow and cavitation in a quasi-superplastic two-phase magnesium-lithium alloy. Materials Science and Engineering A 429: 334-340.

18. Liu XH, Zhan HB, Gu SH, Qu ZK, Wu RZ, et al. (2011) Superplasticity in a two-phase Mg-8Li-2Zn alloy processed by two-pass extrusion. Materials Science and Engineering A 528: 6157-6162.

19.Cao FR, Xia F, Hou HL, Ding H, Li ZQ (2015) Effects of high-density pulse current on mechanical properties and microstructure in a rolled Mg-9.3Li-1.79Al-1.61Zn alloy. Materials Science and Engineering A 637: 89-97.

20.Cao FR, Li ZL, Zhang NX, Ding H, Yu FX, et al. (2013) Superplastity, flow and fracture mechanism in an Al-12.7Si0.7Mg alloy. Materials Science and Engineering A 571: 167-183.

21.Phani KK, Sanyal D (2008) The relations between the shear modulus, the bulk modulus and young's modulus for porous isotropic ceramic materials. Materials Science and Engineering A 490: 305-312.

22.Friedel J (1964) Dislocations. Pergamon Press, Oxford, USA.

23.Chokshi AH (2005) Cavity nucleation and growth in superplasticity. Materials Science and Engineering A 410411: 95-99. 